\title{
Parameterized Schedulability Analysis on Uniform Multiprocessors
}

\author{
Risat Mahmud Pathan and Jan Jonsson \\ Chalmers University of Technology \\ SE-412 96, Göteborg, Sweden \\ \{risat, janjo\}@chalmers.se
}

\begin{abstract}
This paper addresses global rate-monotonic scheduling of implicit-deadline periodic real-time tasks on uniform multiprocessor platforms. In particular, we propose new schedulability conditions that include a set of easily computable task-set parameters for achieving better system utilization while meeting the deadlines of all the tasks. First, an individual sufficient schedulability condition is derived for each task. Then, the collection of schedulability conditions for the tasks are condensed to provide two different simple sufficient schedulability conditions for the entire task system - one for uniform multiprocessors, and one for unit-capacity multiprocessors, respectively. Finally, we show that our proposed simple rate-monotonic schedulability conditions for uniform and unit-capacity multiprocessors have higher worst-case system utilization than all other state-of-the-art simple schedulability conditions for global rate-monotonic scheduling of implicit-deadline tasks.
\end{abstract}

\section{Introduction}

Real-time system applications are often represented using a finite collection of independent recurring processes, for example, in control and monitoring applications. Such recurrent processes are typically modeled using the preemptive periodic task model in which each periodic task's relative deadline is equal to its period (implicit-deadline) [18]. One of the main challenges for such a task model is designing fixed-priority scheduling algorithm that ensures that all the deadlines of the tasks are met during runtime. The fixed-priority Rate-Monotonic (RM) scheduling algorithm [18] is widely used in industry because of its simplicity, flexibility and its ease of implementation [22]. Even though the RM scheduling is optimal for uniprocessor platform, its schedulability performance is poor on multiprocessor platform [13]. Our endeavor in this paper is the design and analysis of RM scheduling to achieve higher system utilization on multiprocessor platform in which the processors may have different speeds (uniform multiprocessors).
Real-time multiprocessor systems use either global scheduling or partitioned scheduling. In global scheduling, a task is allowed to execute on any processor even when it is resumed after preemption. This is done by keeping all the ready tasks in a global queue from which the highestpriority tasks are dispatched to the processors for execution. In partitioned scheduling, the task set is grouped in different task partitions during design time and each partition has a fixed processor onto which only the tasks of that partition are allowed to execute. Many policies for the global [2, 20, 4, 5, 8, 6, 1, 9] and partitioned [13, 21, 7, 3, 19, 17] approaches exist for fixed-priority multiprocessor scheduling. To this end, much research has focused on finding an efficient schedulability condition that, if satisfied, implies that all the deadlines of the tasks are met. In this paper, we present a schedulability analysis (sufficient schedulability condition) for global preemptive RM scheduling for implicit-deadline tasks on uniform multiprocessors and its specialization on unit-capacity multiprocessors.

A system consisting of multiple processors having different computing capacity (speed) is called a uniform multiprocessor platform [15]. On such a platform, a processor with speed $s$ completes $s \times t$ units of execution when a task executes for $t$ time units on the processor. If the speed of each processor is one, then it is a unit-capacity multiprocessor platform. The importance of multiprocessor scheduling on uniform processors has already been pointed out in [14]. In addition, we find two more reasons to study scheduling on uniform multiprocessors. First, when the frequency of different processors' clocks are lowered to save power, the schedulability of the tasks on such a platform can be analyzed using uniform multiprocessor theory. Second, it is very likely that chip multiprocessors [11] will have cores with different speeds in the future.

The quality of many multiprocessor scheduling algorithms is measured in terms of the worst-case system utilization (also known as utilization bound). The worst-case system utilization of a scheduling algorithm is used to provide a sufficient schedulability condition for that algorithm. This is because any set of tasks is guaranteed to be schedu- 
lable (i.e. meet all the deadlines) using a particular scheduling algorithm whenever the total utilization (computation load) of the task set is no larger than the worst-case system utilization. This fact motivates us to design and analyze the RM scheduling algorithm for the multiprocessor platform in order to achieve a high worst-case system utilization.

The main reason for using the total utilization of a task set in a sufficient schedulability condition is that this metric is easy to compute and also represents a simple and condensed task-set parameter in the schedulability condition. In this paper, we introduce three new task-set parameters (formally defined later) that can be easily computed for any task set - the minimum period ratio, the maximum period ratio, and the sum of squares of individual task utilization. Using these parameters, we derive a sufficient schedulability condition for global RM scheduling of implicit-deadline periodic task sets that has a higher worst-case system utilization compared to that of all other similar conditions for global RM scheduling. To this end, we make the following major contributions in this paper:

1. Using the three new task-set parameters (mentioned above), an individual sufficient RM schedulability condition for each task is derived for uniform multiprocessors (i.e. all the sufficient conditions are checked iteratively for all the tasks). These individual conditions are then condensed into two simple (i.e. non-iterative) sufficient RM schedulability conditions for the entire task set - one for uniform multiprocessors and one for unit-capacity multiprocessors.

2. To the best of our knowledge, our simple schedulability conditions for the uniform and unit-capacity multiprocessors dominate all such simple RM schedulability conditions proposed by others for implicit-deadline task systems ${ }^{1}$. However, for other simple fixed-priority schedulability conditions that our simple conditions do not dominate, it is also true that those conditions do not dominate ours either.

3. The three new task-set parameters have an important implication for the system designer in that there exist certain strategies for selecting these task-set parameters so as to achieve a good system performance.

The rest of the paper is organized as follows. First, in Section 2, other works related to multiprocessor scheduling are discussed. In Section 3, the task model used in this work is presented. The model of the multiprocessor platform and some related theory is discussed in Section 4 Then, we present our schedulability analysis for uniform and unitcapacity multiprocessors in Section 5. In Section 6, we compare the performance of our schedulability condition with other works. Finally, Section 7 concludes this paper.

\footnotetext{
${ }^{1}$ By domination we mean that all task sets that satisfy other schedulability conditions must also satisfy our condition, and not vice versa.
}

\section{Related Work}

Fixed-priority multiprocessor scheduling with non-zero worst-case system utilization was first proposed in [2] using a concept called utilization separation. The RM-US algorithm in [2] assigns the highest priority to the tasks having individual task's utilization greater than $\frac{m}{3 m-2}(m$ is the number of processors) while the rest of the tasks are assigned priorities according to RM. It is proved that the worst-case system utilization of algorithm RM-US is $\frac{m^{2}}{3 m-2} \approx 33.33 \%$ on $m$ processors (as $m \rightarrow \infty$ ). The worst-case system utilization of hybrid priority-assignment algorithms such as RM-US has been shown to be as high as approximately $37.48 m \approx 37.48 \%$ [20]. Hybrid priorityassignment based on the slack (i.e. task's period minus the task's execution time) of each task was proposed in [1]. The worst-case system utilization of the SM-US algorithm in [1] is approximately $\frac{2 m}{3+\sqrt{5}} \approx 38.19 \%$ for implicit deadline tasks. Recently, a new fixed-priority assignment scheme for global scheduling was proposed [12], which has been shown (using simulation) to have better performance than other fixed-priority scheduling algorithm.

Based on the minimum amount of interference in an interval that can cause a task's deadline to be missed, a fixedpriority schedulability analysis is given in [4], showing a worst-case system utilization of $\frac{m\left(1-u_{\max }\right)}{2}+u_{\min }$ for RM scheduling on $m$ processors, where $u_{\max }$ and $u_{\min }$ are the maximum and minimum utilization of any task, respectively. RM scheduling on uniform multiprocessors is studied in [5], and it is shown (using a schedulability analysis similar to that of [2]) that the worst-case system utilization is $\frac{m}{3}$ for RM scheduling on $m$ unit-capacity processors.

The work in [8] derives a simple (i.e. non-iterative) sufficient RM schedulability condition that dominates the simple schedulability conditions in [2, 4, 5] for implicit deadline tasks. Using an analysis of the worst-case workload in an interval, the analysis in [8] shows that, if the total utilization of a task set is no larger than $\frac{m\left(1-u_{\max }\right)}{2}+u_{\max }$, then all the tasks deadlines will be met during run time. The work in [8] is further extended and improved in [9], where an iterative algorithm for testing the schedulability of each task separately is proposed. Iterative sufficient scheduling conditions are also derived in [6] for fixed-priority tasks with arbitrary deadlines. Our objective in this paper is to derive simple (i.e. non-iterative) sufficient schedulability conditions, where only one condition has to be verified for the complete task set to determine the RM schedulability on a uniform or unit-capacity multiprocessor platform. Also note that we analyze the schedulability of task sets having traditional RM priority. However, our schedulability analysis can be easily extended to hybrid priority-assignment (similar to [16]) using the concept of utilization-separation. 


\section{Task Model and Related Definitions}

In this paper, we consider the scheduling of $n$ periodic tasks in the set $\left\{\tau_{1}, \tau_{2}, \ldots \tau_{n}\right\}$. Each task $\tau_{i}$ in set $\left\{\tau_{1}, \tau_{2}, \ldots \tau_{n}\right\}$ is characterized by a pair $\left(C_{i}, T_{i}\right)$, where $C_{i}$ represents the worst-case execution time (WCET) and $T_{i}$ is the period (inter-arrival time) of task $\tau_{i}$. Since the task set is periodic, an instance of each task, called job, is released at each period $T_{i}$ and requires at most $C_{i}$ units of execution time before the next period. Thus, the relative deadline of a task $\tau_{i}$ is equal to its period $T_{i}$, that is, the set $\left\{\tau_{1}, \tau_{2}, \ldots \tau_{n}\right\}$ is an implicit-deadline periodic task system.

The fixed priority of the tasks are given according to the RM discipline. In other words, the shorter the task period, the higher the task priority. Without loss of generality, we assume that task $\tau_{i}$ has higher priority than that of task $\tau_{i+1}$, for all $i, 1 \leq i<n$. Therefore, we have $T_{i} \leq T_{j}$ for all $i$ and $j$ such that $1 \leq i \leq j \leq n$.

Since the execution of a task $\tau_{k}$ can be interfered only by the higher-priority tasks using preemptive RM scheduling, whether task $\tau_{k}$ meets its deadline or not depends on the tasks in $\left\{\tau_{1}, \tau_{2}, \ldots \tau_{k}\right\}$ (but are completely unaffected by the tasks in $\left\{\tau_{k+1}, \tau_{k+2}, \ldots \tau_{n}\right\}$.) We find it useful to define the task set $\Gamma^{k} \stackrel{\text { def }}{=}\left\{\tau_{1}, \tau_{2}, \ldots \tau_{k}\right\}$ for $k=1,2 \ldots n$. Note that $\Gamma^{n}=\left\{\tau_{1}, \tau_{2}, \ldots \tau_{n}\right\}$. The collection of all the jobs of all the $k$ tasks in set $\Gamma^{k}$ constitute an implicit-deadline task set $\Gamma^{k}$. We sometimes, hereafter, also use the notation $\Gamma^{k}$ to denote the collection of all the jobs in $\Gamma^{k}$.

Next, we define a couple of task-set parameters, and some important properties of these parameters, that we will use later in this paper.

We define the load or utilization of a task $\tau_{i}$ as $u_{i}=C_{i} / T_{i}$ and the total utilization of the task set $\Gamma^{k}$ as $U^{k}=\sum_{i=1}^{k} u_{i}$ for $k=1,2 \ldots n$. Note that $U^{n}$ is the total utilization of the task set $\Gamma^{n}$.

We define the maximum utilization $u_{\max }^{k}$ of a periodic task system $\Gamma^{k}$ to be the largest utilization of any task in $\Gamma^{k}$ such that $u_{\max }^{k} \stackrel{\text { def }}{=} \max _{\tau_{i} \in \Gamma^{k}} \quad u_{i}$.

Similarly, we define the minimum utilization $u_{m i n}^{k}$ of a periodic task system $\Gamma^{k}$ to be the smallest utilization of any task in $\Gamma^{k}$ such that $u_{\text {min }}^{k} \stackrel{\text { def }}{=} \min _{\tau_{i} \in \Gamma^{k}} \quad u_{i}$.

Note that, for $k \leq n$, we must have

$$
u_{\min }^{n} \leq u_{\min }^{k} \leq u_{\max }^{k} \leq u_{\max }^{n}
$$

We define the minimum period ratio $r_{k}^{\prime}$ of a task set $\Gamma^{k}$ to be the minimum of the ratios of periods of any two tasks $\tau_{i} \in \Gamma^{k}$ and $\tau_{j} \in \Gamma^{k}$ such that $T_{i} \leq T_{j}$ and $i \neq j$. Formally,

$$
r_{k}^{\prime} \stackrel{\text { def }}{=} \min _{1 \leq i<j \leq k}\left\{T_{i} / T_{j}\right\}
$$

Similarly, we define the maximum period ratio $r_{k}^{\prime \prime}$ of a task set $\Gamma^{k}$ to be the maximum of the ratios of periods of any two tasks $\tau_{i} \in \Gamma^{k}$ and $\tau_{j} \in \Gamma^{k}$ such that $T_{i} \leq T_{j}$ and $i \neq j$. Formally,

$$
r_{k}^{\prime \prime} \stackrel{\text { def }}{=} \max _{1 \leq i<j \leq k}\left\{T_{i} / T_{j}\right\}
$$

Note that, using Eq. (3) for any two tasks $\tau_{i} \in \Gamma^{k}$ and $\tau_{j} \in \Gamma^{k}$ such that $T_{i} \leq T_{j}$ and $i \neq j$, we have

$$
\frac{T_{i}}{T_{j}} \leq r_{k}^{\prime \prime} \equiv \frac{T_{i}}{r_{k}^{\prime \prime}} \leq T_{j}
$$

Also note that, for task systems $\Gamma^{k}$ and $\Gamma^{n}$ such that $k \leq n$, we also have

$$
r_{n}^{\prime} \leq r_{k}^{\prime} \leq r_{k}^{\prime \prime} \leq r_{n}^{\prime \prime} \leq 1
$$

We, furthermore, define $\mathrm{Q}^{k}$ as the sum of squares of individual task utilization of the tasks in a task set $\Gamma^{k}$ excluding the task with maximum utilization in $\Gamma^{k}$ by

$$
\mathrm{Q}^{k} \stackrel{\text { def }}{=} \sum_{i=1}^{k} u_{i}^{2}-\left(u_{\max }^{k}\right)^{2}=\sum_{\substack{i=1 \\ i \neq \max \_ \text {index }(k)}}^{k} u_{i}^{2}
$$

such that max_index $(k)$ is the index of the task that has the maximum utilization in the task set $\Gamma^{k}$ for $k=1,2, \ldots n$. Since $\left(u_{\max }^{k}\right)^{2}$ is not included in $\mathrm{Q}^{k}$, we must have

$$
\mathrm{Q}^{k} \leq \sum_{i=1}^{k-1} u_{i}^{2}
$$

And, for $n$, we have the following:

$$
\begin{aligned}
& U^{n}-u_{\max }^{n}-\mathrm{Q}^{n} \geq 0 \\
\equiv & \sum_{\substack{i=1 \\
i \neq \text { max_index }(n)}}^{n} u_{i}-\mathrm{Q}^{n} \geq 0 \\
\equiv & \sum_{\substack{i=1 \\
i \neq \text { max_index }(n)}}^{n} u_{i}-\sum_{\substack{i=1 \\
i \neq \text { max_index }(n)}}^{n} u_{i}^{2} \geq 0 \\
\Leftarrow & \left(\text { From Eq. [6] and the fact that } u_{i}^{2} \leq u_{i} \leq 1 \text { for any } \tau_{i}\right)
\end{aligned}
$$

From Eq. (5), we have $\left(r_{n}^{\prime} \leq r_{n}^{\prime \prime}\right) \Rightarrow\left(\frac{r_{n}^{\prime}}{r_{n}^{\prime \prime}} \leq 1\right)$. Therefore from Eq. (8) we have,

$$
U^{n}-u_{\max }^{n}-\frac{r_{n}^{\prime}}{r_{n}^{\prime \prime}} \cdot \mathrm{Q}^{n} \geq 0
$$

and since $u_{\max }^{n} \geq u_{\min }^{n}$ from Eq. (1), we also have,

$$
U^{n}-u_{m i n}^{n}-\frac{r_{n}^{\prime}}{r_{n}^{\prime \prime}} \cdot \mathrm{Q}^{n} \geq 0
$$

\section{System Model and Related Theory}

The periodic task system defined in Section 3 is to be scheduled on a multiprocessor system. The following three definitions (Definition 1, 2, 3) from [5] are the key concepts, regarding scheduling on uniform multiprocessor platform, that we will use in this paper. 
Definition 1 (from [5]). Let $\pi$ denote a uniform multiprocessor platform.

- The number of processors in $\pi$ is denoted by $m(\pi)$

- For all $i, 1 \leq i \leq m(\pi)$, the speed of the $i^{\text {th }}$ fastest processor in $\pi$ is denoted by $s_{i}(\pi)$, i.e., the speed are indexed in a non-increasing order.

- The total computing capacity of all the processors in $\pi$ is denoted by $S(\pi) \stackrel{\text { def }}{=} \sum_{i=1}^{m(\pi)} s_{i}(\pi)$.

Next we present two useful parameters from [5] that are defined to measure the degree by which uniform multiprocessor platform $\pi$ differs from an identical or unit-capacity multiprocessor platform.

Definition 2 ( $\lambda$ and $\mu$ from [5]). For any uniform multiprocessor platform $\pi$, we define a parameter $\lambda(\pi)$ as follows:

$$
\lambda(\pi) \stackrel{\text { def }}{=} \underset{i=1}{m a x}\left\{\frac{\sum_{j=i+1}^{m(\pi)} s_{j}(\pi)}{s_{i}(\pi)}\right\}
$$

For any uniform multiprocessor platform $\pi$, we define a parameter $\mu(\pi)$ as follows:

$$
\mu(\pi) \stackrel{\text { def }}{=} \underset{i=1}{m(\pi)}\left\{\frac{\sum_{j=i}^{m(\pi)} s_{j}(\pi)}{s_{i}(\pi)}\right\}
$$

If the speeds of the processors differs from each other significantly, we have $\lambda(\pi)=0$ and $\mu(\pi)=1$, and if we are considering identical or unit-capacity multiprocessor platform, we have $\lambda(\pi)=(m(\pi)-1)$ and $\mu(\pi)=m(\pi)$. Note that, the relationship between $\mu(\pi)$ and $\lambda(\pi)$ is as follows.

$$
\mu(\pi)=1+\lambda(\pi)
$$

Scheduling algorithm on uniform multiprocessor requires a stronger notion of work-conserving algorithm (defined below), called greedy scheduling algorithms, that is defined next according to [5].

Definition 3 (Greedy Scheduling Algorithm). A uniform multiprocessor scheduling is greedy if it satisfies all three of the following conditions.

1. It is work conserving, that is, it never idles a processor when there are jobs awaiting execution.

2. If it must idle some processor (fewer active jobs than processors), then it idles the slowest processor.

3. It always executes higher priority jobs upon faster processors. If job $J_{1}$ and $J_{2}$ are executing on the $i^{\text {th }}$ and $j^{\text {th }}$ processors for $i<j$, then job $J_{1}$ 's priority is higher than the priority of job $J_{2}$.

In rest of the paper, we consider that the global scheduling algorithm RM is greedy. Our objective in this paper is to analyze the RM schedulability of a task set $\Gamma^{n}$ on a uniform multiprocessor platform $\pi$. To characterize the amount of work done by any scheduling algorithm $A$ on a uniform multiprocessor platform $\pi$ over a time interval of length $t$, we have the following well-known definition of work [5].
Definition $4\left(W\left(A, \pi, \Gamma^{k}, t\right)\right)$. Let any uniform multiprocessor platform $\pi$ on which the task set $\Gamma^{k}$ is to be executed using any algorithm $A$. For time instant $t \geq 0$, let $W\left(A, \pi, \Gamma^{k}, t\right)$ denote the amount of work done by algorithm $A$ on jobs of the task set $\Gamma^{k}$ over the interval $[0, t)$, while executing on $\pi$.

The following Theorem 1 (proved in [14]) provides a lower bound on the amount of work completed by a greedy scheduling algorithm $A$ on a uniform multiprocessor platform $\pi$, in terms of the amount of work completed by algorithm $A_{0}$ on a uniform multiprocessor platform $\pi_{0}$, when the inequality in Eq. (12) is satisfied for the platforms $\pi$ and $\pi_{0}$. We will be using Theorem 1 later in this section.

Theorem 1 (From [14]). Let $\pi_{0}$ and $\pi$ denote uniform multiprocessor platform. Let $A_{0}$ denote any uniform multiprocessor scheduling algorithm, and A any greedy uniform multiprocessor scheduling algorithm. If the following condition is satisfied by platforms $\pi$ and $\pi_{0}$ :

$$
S(\pi) \geq S\left(\pi_{0}\right)+\lambda(\pi) \cdot s_{1}\left(\pi_{0}\right)
$$

then, for any collection of jobs of task set $\Gamma^{k}$ and any time instant $t \geq 0$,

$$
W\left(A, \pi, \Gamma^{k}, t\right) \geq W\left(A_{0}, \pi_{0}, \Gamma^{k}, t\right)
$$

It will be evident later that the schedulability analysis presented in Section 5 is based on the lower and upper bounds of the amount of work completed within a particular time interval by the greedy RM scheduling on a uniform multiprocessor platform $\pi$. Next we present the results of some previous works that estimate the lower and upper bound of the amount of work completed by the RM scheduling algorithm.

\subsection{Lower Bound on Work}

In this subsection we calculate the lower bound on the amount of work done within a time interval $[0, t)$ by the greedy RM scheduling on jobs of the task set $\Gamma^{k}$. The following Lemma 1 (proved in [5]) shows that there exists a uniform multiprocessor platform $\pi_{0}$ on which a task system $\Gamma^{k}$ is schedulable.

Lemma 1 (From [5]). Task system $\Gamma^{k}$ is feasible on a uniform multiprocessor platform $\pi_{0}$ satisfying the conditions:

$$
S\left(\pi_{0}\right)=U^{k} \text { and } s_{1}\left(\pi_{0}\right)=u_{\max }^{k}
$$

The proof of Lemma 1 is based on the construction of the uniform multiprocessor platform $\pi_{0}$ with $k$ processors with speed $s_{1}\left(\pi_{0}\right), s_{2}\left(\pi_{0}\right) \ldots s_{k}\left(\pi_{0}\right)$ such that $s_{i}\left(\pi_{0}\right)=u_{i}$ and $u_{i}$ is the utilization of the task $\tau_{i} \in \Gamma^{k}$. Note that, such a platform must satisfy $S\left(\pi_{0}\right)=U^{k}$ and $s_{1}\left(\pi_{0}\right)=u_{\max }^{k}$ for the task set $\Gamma^{k}$ for $k=1,2, \ldots n$. The task set $\Gamma^{k}$ is schedulable on the platform $\pi_{0}$ using the scheduling algorithm, called OP T, that exclusively executes the task $\tau_{i} \in \Gamma^{k}$ on the processor with speed $s_{i}$. 
According to the definition of uniform multiprocessor, a task $\tau_{i}$ executes on processor with speed $s_{i}$ for $t$ time units completes $t \times s_{i}$ units of execution. Therefore, the amount of work completed within the interval $[0, t)$ by the scheduling algorithm OP T on the jobs of the task set $\Gamma^{k}$ on platform $\pi_{0}$ is given by Eq. (14).

$$
W\left(\mathrm{OPT}, \pi_{0}, \Gamma^{k}, t\right)=\sum_{i=1}^{k} t \cdot s_{i}=t \cdot \sum_{i=1}^{k} u_{i}
$$

Recall that we want to execute a task set on uniform multiprocessor platform $\pi$ using greedy RM scheduling. If the uniform multiprocessor platforms $\pi$ and $\pi_{0}$ satisfies the condition in Eq. (12) of Theorem 1 (where $\pi_{0}$ is the platform guaranteed to exist according to Lemma 11) and $\mathrm{RM}$ is greedy scheduling, then using inequality Eq. [13) of Theorem 1 we have

$$
W\left(\mathrm{RM}, \pi, \Gamma^{k}, t\right) \geq W\left(\mathrm{OPT}, \pi_{0}, \Gamma^{k}, t\right)=t \cdot \sum_{i=1}^{k} u_{i}
$$

According to Eq. [15, the amount of work completed over the interval $[0, t)$ by the RM scheduling on the jobs of the task set $\Gamma^{k}$ while executing on platform $\pi$ is lower bounded by $t \cdot \sum_{i=1}^{k} u_{i}$ if the inequality in Eq. (12) is satisfied for platforms $\pi$ and $\pi_{0}$. It is worth mentioning at this point that Eq. (15) is originally derived in [5] by showing that Eq. (12) is always implied by the sufficient RM schedulability condition that is proposed in [5].

\subsection{Upper Bound on Work}

We now derive an upper bound on the total amount of maximum work that can be completed by the jobs of the tasks in set $\left\{\tau_{1}, \tau_{2}, \ldots \tau_{k-1}\right\}$ over time interval $(0, t]$.

Over the interval $[0, t)$, exactly $\left\lfloor\frac{t}{T_{i}}\right\rfloor$ complete jobs of task $\tau_{i}$ are scheduled and the $\left(\left\lfloor\frac{t}{T_{i}}\right\rfloor+1\right)^{t h}$ job of $\tau_{i}$ may be scheduled for at most $\min \left(t-\left\lfloor\frac{t}{T_{i}}\right\rfloor T_{i}, C_{i}\right)$ time units. The maximum amount of work completed by the jobs of task $\tau_{i}$ within any time interval $[0, t)$, which is at most $\left\lfloor\frac{t}{T_{i}}\right\rfloor C_{i}+\min \left(t-\left\lfloor\frac{t}{T_{i}}\right\rfloor T_{i}, C_{i}\right)$, is upper bounded using the following Lemma 2 that is proved in [1, 10].

Lemma 2 (From [1, 10]). The maximum amount of work $\left\lfloor\frac{t}{T_{i}}\right\rfloor C_{i}+\min \left(t-\left\lfloor\frac{t}{T_{i}}\right\rfloor T_{i}, C_{i}\right)$ that can be completed by task $\tau_{i}$ over an interval $[0, t)$ is upper bounded by the following inequality in Eq. (16)

$$
\begin{aligned}
&\left\lfloor\frac{t}{T_{i}}\right\rfloor C_{i}+ \min \left(t-\left\lfloor\frac{t}{T_{i}}\right\rfloor T_{i}, C_{i}\right) \\
& \leq C_{i}+\left(t-C_{i}\right) \frac{C_{i}}{T_{i}}
\end{aligned}
$$

By summing the Eq. (16) over $i=1,2, \ldots(k-1)$, the maximum amount of work completed by the jobs of the tasks in set $\left\{\tau_{1}, \tau_{2}, \ldots \tau_{k-1}\right\}$ over the time interval $(0, t]$ is upper bounded by the right-hand side of the following inequality in Eq. 17)

$$
\begin{aligned}
\sum_{i=1}^{k-1}\left[\left\lfloor\frac{t}{T_{i}}\right\rfloor C_{i}\right. & \left.+\min \left(t-\left\lfloor\frac{t}{T_{i}}\right\rfloor T_{i}, C_{i}\right)\right] \\
\leq & \sum_{i=1}^{k-1}\left[C_{i}+\left(t-C_{i}\right) \frac{C_{i}}{T_{i}}\right]
\end{aligned}
$$

Next we present our global RM schedulability analysis on uniform multiprocessors based on the inequalities given in Eq. 15) and Eq. 177.

\section{Schedulability Analysis}

In this section, we derive a sufficient schedulability condition for global RM scheduling on uniform multiprocessor platform. First, the maximum available time for executing the $l^{\text {th }}$ job of task $\tau_{k}$ is calculated using the difference between the upper and lower bound on work completed by the RM scheduling over time intervals $\left(0, l T_{k}\right]$ and $\left(0,(l-1) T_{k}\right]$, respectively. Based on this difference, a sufficient schedulability condition is derived for task $\tau_{k}$ in Subsection 5.1 for $k=1,2 \ldots n$ (resulting in an iterative sufficient schedulability condition for the entire task set). Then in Subsection 5.2 the schedulability conditions of all the tasks are condensed into a simple (non-iterative) sufficient schedulability condition for uniform multiprocessors. Based on the simple sufficient schedulability condition derived in Subsection 5.2 for uniform multiprocessors, a corresponding simple suffient schedulability condition is derived for unit-capacity multiprocessors in Subsection 5.3 .

\subsection{Iterative Schedulability Condition for $\pi$}

In this subsection, a sufficient RM schedulability condition of the task set $\Gamma^{n}$ on uniform multiprocessor platform $\pi$ is derived using the sufficient RM schedulability condition of each task $\tau_{k}$, for $k=1,2, \ldots n$. The following Lemma 3 proves that all the jobs of task $\tau_{k}$ meet their deadlines using global RM scheduling on platform $\pi$ if the conditions in Eq. (18) and Eq. (19) are satisfied (a sufficient schedulability condition for task $\tau_{k}$ ).

Lemma 3. All the jobs of $\tau_{k}$ meet their deadlines when $\Gamma^{k}$ is scheduled on $\pi$ using global RM scheduling if the two following conditions are satisfied:

$$
S(\pi) \geq U^{k}+\lambda(\pi) \cdot u_{\max }^{k}
$$

and,

$$
\left[\frac{S(\pi)-\mu(\pi) \cdot u_{k}}{\left(1+r_{k}^{\prime \prime}\right)}+u_{k}+\frac{r_{k}^{\prime \prime} \cdot \mathrm{Q}^{k}}{\left(1+r_{k}^{\prime \prime}\right)}\right] \geq U^{k}
$$

Proof. Lets assume that all the $(l-1)$ jobs of $\tau_{k}$ have met their deadlines using RM. We will prove that the $l^{\text {th }}$ job of $\tau_{k}$ also meets the deadline. The correctness of Lemma 3 will then follow by induction on $l \geq 1$. 
Since each job of task $\tau_{k}$ is released at each period $T_{k}$, the $l^{t h}$ job arrives at time $(l-1) T_{k}$ and requires $C_{k}$ units of execution time before its deadline $l T_{k}$. Remember that task set $\Gamma^{k}$ is schedulable using algorithm OPT on uniform multiprocessor platform $\pi_{0}$ (Lemma 1 ). The platform $\pi_{0}$, that is shown to exist by construction in the discussion following Lemma11 must satisfy $S\left(\pi_{0}\right)=U^{k}$ and $s_{1}\left(\pi_{0}\right)=u_{\max }^{k}$. It follows from Eq. (18) that $S(\pi) \geq S\left(\pi_{0}\right)+\lambda(\pi) \cdot s_{1}\left(\pi_{0}\right)$ is true for the uniform multiprocessor platforms $\pi$ and $\pi_{0}$. Therefore, the condition in Eq. (12) of Theorem 1 is true whenever the condition in Eq. (18) is true. Since the condition in Eq. (12) is true, using Eq. (15) we have

$$
\begin{gathered}
W\left(\mathrm{RM}, \pi, \Gamma^{k},(l-1) T_{k}\right) \geq(l-1) T_{k} \sum_{i=1}^{k} u_{i}= \\
(l-1) T_{k} \sum_{i=1}^{k-1} u_{i}+(l-1) T_{k} u_{k}
\end{gathered}
$$

According to Eq. 200, the minimum amount of work completed by RM before the $l^{\text {th }}$ job of $\tau_{k}$ arrives at time $(l-1) T_{k}$ is $(l-1) T_{k} \quad \sum_{i=1}^{k-1} u_{i}+(l-1) T_{k} u_{k}$. Note that, prior to time instant $(l-1) T_{k}$, the amount of work generated for task $\tau_{k}$ is exactly $(l-1) T_{k} u_{k}$. Since we assume that all the $(l-1)$ jobs of task $\tau_{k}$ have met their deadlines (inductive hypothesis), the total work executed by $\mathrm{RM}$ for the higher-priority tasks $\tau_{1}, \tau_{2}, \ldots \tau_{k-1}$ is at least $(l-1) T_{k} \sum_{i=1}^{k-1} u_{i}$ prior to the time instant $(l-1) T_{k}$.

Lemma 2 ensures that the maximum amount of work that can be completed by all the higher-priority tasks $\tau_{1}, \tau_{2}, \ldots \tau_{k-1}$ over the interval $\left[0, l T_{k}\right)$ is bounded from above by $\sum_{i=1}^{k-1}\left[C_{i}+\left(l T_{k}-C_{i}\right) \frac{C_{i}}{T_{i}}\right]$. In the previous paragraph, we saw that at least $(l-1) T_{k} \sum_{i=1}^{k-1} u_{i}$ of this work is completed prior to time instant $(l-1) T_{k}$. Therefore, at most

$$
\begin{array}{r}
\sum_{i=1}^{k-1}\left[\left(C_{i}+\left(l T_{k}-C_{i}\right) u_{i}\right)-\left((l-1) T_{k} u_{i}\right)\right] \\
=\sum_{i=1}^{k-1}\left(C_{i}+\left(T_{k}-C_{i}\right) u_{i}\right)
\end{array}
$$

amount of work remains to be executed after time instant $(l-1) T_{k}$ for all the higher priority tasks $\tau_{1}, \tau_{2}, \ldots \tau_{k-1}$.

The amount of processors capacity left unused by tasks $\tau_{1}, \tau_{2}, \ldots \tau_{k-1}$ during the interval $\left[(l-1) T_{k}, l T_{k}\right)$ on the uniform multiprocessor platform $\pi$ is therefore at least

$$
S(\pi) \cdot T_{k}-\sum_{i=1}^{k-1}\left(C_{i}+\left(T_{k}-C_{i}\right) u_{i}\right)
$$

Not all of this capacity is available to the $l^{\text {th }}$ job of $\tau_{k}$ if several processors are available at the same time. In the worst-case, if all the $m(\pi)$ processors are available at the same time, the $l^{t h}$ job of $\tau_{k}$ can execute only on one processor. Since RM is greedy, if several processors are simultaneously available, then $\tau_{k}$ will execute upon the fastest available processor (Definition 3 ). If $\tau_{k}$ executes on the $j^{t h}$ fastest processor at some instant, then all other processors with speed $s_{x}(\pi)$ for $x<j$ are busy at this instant and does not contribute to the remaining capacity given in Eq. (21). Therefore, the fraction of total remaining capacity given in Eq. (21) that can be used for executing the $l^{t h}$ job of task $\tau_{k}$ at this instant is at least $\frac{s_{j}(\pi)}{\left[s_{j}(\pi)+s_{j+1}(\pi)+\ldots+s_{m(\pi)}(\pi)\right]}$. This reasoning is similar to that of used in [5]. According to Definition 2, we have $\frac{s_{j}(\pi)}{\left[s_{j}(\pi)+s_{j+1}(\pi)+\ldots+s_{m(\pi)}(\pi)\right]} \geq \frac{1}{\mu(\pi)}$. Consequently, the amount of processing capacity available to the $l^{t h}$ job of $\tau_{k}$ during the interval $\left[(l-1) T_{k}, l T_{k}\right)$ on the uniform multiprocessor platform $\pi$ is at least

$$
\frac{1}{\mu(\pi)}\left[S(\pi) \cdot T_{k}-\sum_{i=1}^{k-1}\left(C_{i}+\left(T_{k}-C_{i}\right) u_{i}\right)\right]
$$

To guarantee that the $l^{\text {th }}$ job of $\tau_{k}$ meets its deadline, we need this capacity to be at least as large as the execution time of $\tau_{k}$; that is, we must have,

$$
\begin{aligned}
& \frac{1}{\mu(\pi)}\left[S(\pi) \cdot T_{k}-\sum_{i=1}^{k-1}\left(C_{i}+\left(T_{k}-C_{i}\right) u_{i}\right)\right] \geq C_{k} \\
& \equiv \frac{1}{\mu(\pi)}\left[S(\pi) \cdot T_{k}-\sum_{i=1}^{k-1}\left(C_{i}+\frac{T_{k} C_{i}}{T i}-\frac{C_{i}^{2}}{T_{i}}\right)\right] \geq C_{k} \\
& \equiv \frac{1}{\mu(\pi)}\left[S(\pi)-\sum_{i=1}^{k-1}\left(\frac{C_{i}}{T_{k}}+\frac{C_{i}}{T_{i}}-\frac{C_{i}^{2}}{T_{i} T_{k}}\right)\right] \geq \frac{C_{k}}{T_{k}} \\
& \equiv \frac{1}{\mu(\pi)}\left[S(\pi)-\sum_{i=1}^{k-1}\left(\frac{C_{i}}{T_{i}}+\frac{C_{i}}{T_{k}}\left(1-\frac{C_{i}}{T_{i}}\right)\right)\right] \geq \frac{C_{k}}{T_{k}} \\
& \Leftarrow\left(\text { Since } T_{i} \leq T_{k} \text {, using Eq. (4), we have } \frac{T_{i}}{r_{k}^{\prime \prime}} \leq T_{k}\right) \\
& \frac{1}{\mu(\pi)}\left[S(\pi)-\sum_{i=1}^{k-1}\left(\frac{C_{i}}{T_{i}}+\frac{r_{k}^{\prime \prime} \cdot C_{i}}{T_{i}}\left(1-\frac{C_{i}}{T_{i}}\right)\right)\right] \geq \frac{C_{k}}{T_{k}} \\
& \equiv \frac{1}{\mu(\pi)}\left[S(\pi)-\left(1+r_{k}^{\prime \prime}\right) \sum_{i=1}^{k-1} \frac{C_{i}}{T_{i}}+r_{k}^{\prime \prime} \sum_{i=1}^{k-1} \frac{C_{i}^{2}}{T_{i}^{2}}\right] \geq \frac{C_{k}}{T_{k}} \\
& \equiv \frac{1}{\mu(\pi)}\left[S(\pi)-\left(1+r_{k}^{\prime \prime}\right) \sum_{i=1}^{k-1} u_{i}+r_{k}^{\prime \prime} \sum_{i=1}^{k-1} u_{i}^{2}\right] \geq u_{k} \\
& \equiv S(\pi)-\left(1+r_{k}^{\prime \prime}\right) \sum_{i=1}^{k-1} u_{i}+r_{k}^{\prime \prime} \sum_{i=1}^{k-1} u_{i}^{2} \geq \mu(\pi) \cdot u_{k} \\
& \equiv S(\pi)-\left(1+r_{k}^{\prime \prime}\right)\left(U^{k}-u_{k}\right)+r_{k}^{\prime \prime} \sum_{i=1}^{k-1} u_{i}^{2} \geq \mu(\pi) \cdot u_{k} \\
& \equiv S(\pi)-\mu(\pi) \cdot u_{k}+\left(1+r_{k}^{\prime \prime}\right) u_{k}+r_{k}^{\prime \prime} \sum_{i=1}^{k-1} u_{i}^{2} \geq\left(1+r_{k}^{\prime \prime}\right) \cdot U^{k} \\
& \equiv \frac{S(\pi)-\mu(\pi) \cdot u_{k}}{\left(1+r_{k}^{\prime \prime}\right)}+u_{k}+\frac{r_{k}^{\prime \prime}}{\left(1+r_{k}^{\prime \prime}\right)} \sum_{i=1}^{k-1} u_{i}^{2} \geq U^{k} \\
& \Leftarrow\left(\text { Since } \mathrm{Q}^{k} \leq \sum_{i=1}^{k-1} u_{i}^{2}\right. \text { using Eq. (7) } \\
& {\left[\frac{S(\pi)-\mu(\pi) \cdot u_{k}}{\left(1+r_{k}^{\prime \prime}\right)}+u_{k}+\frac{r_{k}^{\prime \prime} \cdot \mathrm{Q}^{k}}{\left(1+r_{k}^{\prime \prime}\right)}\right] \geq U^{k}}
\end{aligned}
$$$$
\Leftarrow(\text { Premise in Eq. 19] of this Lemma) }
$$ 
Next the sufficient RM schedulability condition of the entire task set $\Gamma^{n}$ is given in Theorem 2 based on the sufficient schedulability condition in Lemma 3 .

Theorem 2. An implicit deadline periodic task set $\Gamma^{n}$ is schedulable using RM algorithm on a uniform multiprocessor platform $\pi$, if the following $(n+1)$ conditions are satisfied:

$$
S(\pi) \geq U^{n}+\lambda(\pi) \cdot u_{\max }^{n}
$$

and, for all $k=1,2, \ldots n$,

$$
\left[\frac{S(\pi)-\mu(\pi) \cdot u_{k}}{\left(1+r_{k}^{\prime \prime}\right)}+u_{k}+\frac{r_{k}^{\prime \prime} \cdot \mathrm{Q}^{k}}{\left(1+r_{k}^{\prime \prime}\right)}\right] \geq U^{k}
$$

Proof. If $S(\pi) \geq U^{n}+\lambda(\pi) \cdot u_{\text {max }}^{n}$, then $S(\pi) \geq U^{k}+$ $\lambda(\pi) \cdot u_{\max }^{k}$ because $U^{n} \geq U^{k}$ and $u_{\max }^{n} \geq u_{\max }^{k}$ for all $k=1,2, \ldots n$. According to Eq. (23), we also have that the condition $\left[\frac{S(\pi)-\mu(\pi) \cdot u_{k}}{\left(1+r_{k}^{\prime \prime}\right)}+u_{k}+\frac{r_{k}^{\prime \prime} \cdot \mathrm{Q}^{k}}{\left(1+r_{k}^{\prime \prime}\right)}\right] \geq U^{k}$ is true for all $k=1,2, \ldots n$. Therefore, the two conditions in Eq. (18) and Eq. (19) of Lemma 3 are true for all tasks $\tau_{k}$ for $k=1,2 \ldots, n$. Consequently, all the jobs of all the tasks in set $\Gamma^{n}$ meet their deadlines using RM scheduling on a uniform multiprocessor platform $\pi$ using Lemma3.

Theorem 2 provides a sufficient RM schedulability condition that has to be checked iteratively for all the $n$ tasks in task set $\Gamma^{n}$. Next we derive a simple sufficient RM schedulability condition for $\Gamma^{n}$.

\subsection{Simple Schedulability Condition for $\pi$}

In this subsection, we condense the set of $(n+1)$ conditions given in Theorem 2 to derive a simple sufficient schedulability condition in the sense that it is non-iterative and has to be checked only once for the entire task set $\Gamma^{n}$. We derive the simple sufficient schedulability condition in this subsection such that it implies all the $(n+1)$ conditions given in Eq. (22) and Eq. (23) of Theorem 2 Hence by the transitivity property of implication, if the simple condition is true, then the task set $\Gamma^{n}$ is RM schedulable on platform $\pi$. The simple sufficient feasibility condition for uniform multiprocessor will be given in Theorem 3 Before Theorem 3 is presented, we need the following Lemma 4

Lemma 4. For a non-negative constant $c \leq 1$, the following inequality holds.

$$
U^{n}-c \cdot \mathrm{Q}^{n} \geq U^{k}-c \cdot \mathrm{Q}^{k}
$$

Proof. We start with the following equality using Eq. (6)

$$
U^{n}-c \cdot \mathrm{Q}^{n}=\sum_{i=1}^{k} u_{i}+\sum_{i=k+1}^{n} u_{i}-c \cdot\left[\sum_{i=1}^{n} u_{i}^{2}-\left(u_{\max }^{n}\right)^{2}\right]
$$

Equivalently,

$$
\begin{aligned}
U^{n}-c \cdot \mathrm{Q}^{n} & =\sum_{i=1}^{k} u_{i}+\sum_{i=k+1}^{n} u_{i} \\
& -c \cdot\left[\sum_{i=1}^{k} u_{i}^{2}+\sum_{i=k+1}^{n} u_{i}^{2}-\left(u_{\max }^{n}\right)^{2}\right]
\end{aligned}
$$

Since $c \leq 1$, and $u_{i} \geq u_{i}^{2}$, we have $\sum_{i=k+1}^{n} u_{i} \geq c \cdot \sum_{i=k+1}^{n} u_{i}^{2}$. Therefore from Eq. 25], we have

$$
U^{n}-c \cdot \mathbf{Q}^{n} \geq \sum_{i=1}^{k} u_{i}-c \cdot\left[\sum_{i=1}^{k} u_{i}^{2}-\left(u_{\max }^{n}\right)^{2}\right]
$$

Since $\mathrm{Q}^{k}=\sum_{i=1}^{k} u_{i}^{2}-\left(u_{\max }^{k}\right)^{2}$ according to Eq. (6), we have

$$
\begin{gathered}
U^{n}-c \cdot \mathbf{Q}^{n} \geq \sum_{i=1}^{k} u_{i}-c \cdot\left[\mathbf{Q}^{k}+\left(u_{\max }^{k}\right)^{2}-\left(u_{\max }^{n}\right)^{2}\right] \\
\equiv U^{n}-c \cdot \mathbf{Q}^{n} \geq \sum_{i=1}^{k} u_{i}-c \cdot \mathbf{Q}^{k}+c \cdot\left[\left(u_{\max }^{n}\right)^{2}-\left(u_{\max }^{k}\right)^{2}\right]
\end{gathered}
$$

Since $\left(u_{\max }^{n}\right)^{2} \geq\left(u_{\max }^{k}\right)^{2}$, we have

$$
\begin{aligned}
& U^{n}-c \cdot \mathrm{Q}^{n} \geq \sum_{i=1}^{k} u_{i}-c \cdot \mathrm{Q}^{k} \\
\equiv & U^{n}-c \cdot \mathrm{Q}^{n} \geq U^{k}-c \cdot \mathrm{Q}^{k}
\end{aligned}
$$

We now derive the simple (non-iterative) sufficient condition for RM schedulability of a task set $\Gamma^{n}$ on a uniform multiprocessor platform $\pi$ in Theorem 3

Theorem 3. The task system $\Gamma^{n}$ is RM schedulable on uniform multiprocessor platform $\pi$ if

$$
\frac{S(\pi)-\mu(\pi) \cdot u_{\max }^{n}}{\left(1+r_{n}^{\prime \prime}\right)}+\delta+\frac{r_{n}^{\prime} \cdot \mathrm{Q}^{n}}{\left(1+r_{n}^{\prime \prime}\right)} \geq U^{n}
$$

where

$$
\delta= \begin{cases}u_{\max }^{n} & \text { if } \mu(\pi)>1+r_{n}^{\prime \prime} \\ u_{\min }^{n} & \text { otherwise }\end{cases}
$$

Proof. We prove this theorem by showing that the condition in Eq. (26) implies the following $(n+1)$ conditions:

$$
S(\pi) \geq U^{n}+\lambda(\pi) \cdot u_{\max }^{n}
$$

and, for all $k=1,2, \ldots n$,

$$
\left[\frac{S(\pi)-\mu(\pi) \cdot u_{k}}{\left(1+r_{k}^{\prime \prime}\right)}+u_{k}+\frac{r_{k}^{\prime \prime} \cdot \mathrm{Q}^{k}}{\left(1+r_{k}^{\prime \prime}\right)}\right] \geq U^{k}
$$

given in Theorem 2. We prove these $(n+1)$ conditions of Eq. (22) and Eq. 23) below in Case (1) and Case (2), respectively, based on Eq. (26). Then the RM schedulability of $\Gamma^{n}$ on platform $\pi$ follows from Theorem 2 .

Case (1): We start with the premise of this Theorem given in Eq. 26)

$$
\frac{S(\pi)-\mu(\pi) \cdot u_{\max }^{n}}{\left(1+r_{n}^{\prime \prime}\right)}+\delta+\frac{r_{n}^{\prime} \cdot \mathrm{Q}^{n}}{\left(1+r_{n}^{\prime \prime}\right)} \geq U^{n}
$$

Using Eq. 11, that is $\mu(\pi)=\lambda(\pi)+1$, we have 


$$
\begin{aligned}
& \frac{S(\pi)-[\lambda(\pi)+1] \cdot u_{\max }^{n}}{\left(1+r_{n}^{\prime \prime}\right)}+\delta+\frac{r_{n}^{\prime} \cdot \mathrm{Q}^{n}}{\left(1+r_{n}^{\prime \prime}\right)} \geq U^{n} \\
& \equiv S(\pi)-[\lambda(\pi)+1] \cdot u_{\max }^{n}+\delta \cdot\left(1+r_{n}^{\prime \prime}\right) \\
& +r_{n}^{\prime} \cdot \mathrm{Q}^{n} \geq U^{n} \cdot\left(1+r_{n}^{\prime \prime}\right) \\
& \equiv S(\pi)-\lambda(\pi) \cdot u_{\max }^{n}-u_{\max }^{n}+\delta \geq \\
& U^{n}+r_{n}^{\prime \prime}\left(U^{n}-\delta-\frac{r_{n}^{\prime}}{r_{n}^{\prime \prime}} \cdot \mathrm{Q}^{n}\right)
\end{aligned}
$$

We have $\left(U^{n}-\delta-\frac{r_{n}^{\prime}}{r_{n}^{\prime \prime}} \cdot \mathrm{Q}^{n}\right) \geq 0$ for $\delta=u_{\text {max }}^{n}$ or $\delta=$ $u_{\min }^{n}$ using Eq. (9) or Eq. (10), respectively. Therefore, from Eq. 27, we have

$$
S(\pi)-\lambda(\pi) \cdot u_{\max }^{n}-\left(u_{\max }^{n}-\delta\right) \geq U^{n}
$$

Since $\delta=u_{\max }^{n}$ or $\delta=u_{\min }^{n}$, we have $\left(u_{\max }^{n}-\delta\right) \geq 0$. Therefore,

$$
S(\pi)-\lambda(\pi) \cdot u_{\max }^{n} \geq U^{n}
$$

Since this equation is equivalent to $S(\pi) \geq U^{n}+\lambda(\pi) \cdot u_{\max }^{n}$, the premise of this Theorem implies the first condition Eq. (22) of Theorem 2

Case (2): We have (premise of this theorem)

$$
\begin{aligned}
& \frac{S(\pi)-\mu(\pi) \cdot u_{\max }^{n}}{\left(1+r_{n}^{\prime \prime}\right)}+\delta+\frac{r_{n}^{\prime} \cdot \mathrm{Q}^{n}}{\left(1+r_{n}^{\prime \prime}\right)} \geq U^{n} \\
& \left.\Rightarrow \text { (Using Eq. (5) we have } r_{k}^{\prime \prime} \leq r_{n}^{\prime \prime}\right) \\
& \frac{S(\pi)-\mu(\pi) \cdot u_{\max }^{n}}{\left(1+r_{k}^{\prime \prime}\right)}+\delta+\frac{r_{n}^{\prime} \cdot \mathrm{Q}^{n}}{\left(1+r_{k}^{\prime \prime}\right)} \geq U^{n} \\
& \Rightarrow \text { (Using Eq. (5) we have } r_{n}^{\prime} \leq r_{k}^{\prime \prime} \text {, therefore) } \\
& \frac{S(\pi)-\mu(\pi) \cdot u_{\max }^{n}}{\left(1+r_{k}^{\prime \prime}\right)}+\delta+\frac{r_{k}^{\prime \prime} \cdot \mathrm{Q}^{n}}{\left(1+r_{k}^{\prime \prime}\right)} \geq U^{n} \\
& \equiv \frac{S(\pi)-\mu(\pi) \cdot u_{\max }^{n}}{\left(1+r_{k}^{\prime \prime}\right)}+\delta \geq U^{n}-\frac{r_{k}^{\prime \prime} \cdot \mathrm{Q}^{n}}{\left(1+r_{k}^{\prime \prime}\right)}
\end{aligned}
$$

Since $\frac{r_{k}^{\prime \prime}}{\left(1+r_{k}^{\prime \prime}\right)} \leq 1$, using Eq. 24) of Lemma 4 we have

$$
\left[U^{n}-\frac{r_{k}^{\prime \prime}}{\left(1+r_{k}^{\prime \prime}\right)} \cdot \mathrm{Q}^{n}\right] \geq\left[U^{k}-\frac{r_{k}^{\prime \prime}}{\left(1+r_{k}^{\prime \prime}\right)} \cdot \mathrm{Q}^{k}\right]
$$

Therefore, from Eq. (28) using Eq. (29) we have

$$
\begin{aligned}
& \frac{S(\pi)-\mu(\pi) \cdot u_{\max }^{n}}{\left(1+r_{k}^{\prime \prime}\right)}+\delta \geq U^{k}-\frac{r_{k}^{\prime \prime} \cdot \mathrm{Q}^{k}}{\left(1+r_{k}^{\prime \prime}\right)} \\
\equiv & \frac{S(\pi)}{\left(1+r_{k}^{\prime \prime}\right)}+\delta-\frac{\mu(\pi) \cdot u_{\max }^{n}}{\left(1+r_{k}^{\prime \prime}\right)} \geq U^{k}-\frac{r_{k}^{\prime \prime} \cdot \mathrm{Q}^{k}}{\left(1+r_{k}^{\prime \prime}\right)}
\end{aligned}
$$

The sufficient schedulability condition of this Theorem depends on the value of $\delta$ which can be $u_{\max }^{n}$ or $u_{\min }^{n}$ for $\mu(\pi)>1+r_{n}^{\prime \prime}$ or $\mu(\pi) \leq 1+r_{n}^{\prime \prime}$, respectively. To that end, we have Subcase (2a) and Subcase (2b).

Subcase (2a): In this subcase, we consider $\mu(\pi)>1+r_{n}^{\prime \prime}$ and hence $\delta=u_{\max }^{n}$ according to Eq. (26). From Eq. (30), using $\delta=u_{\max }^{n}$ we have

$$
\frac{S(\pi)}{\left(1+r_{k}^{\prime \prime}\right)}+u_{\max }^{n}\left[1-\frac{\mu(\pi)}{\left(1+r_{k}^{\prime \prime}\right)}\right] \geq U^{k}-\frac{r_{k}^{\prime \prime} \cdot \mathrm{Q}^{k}}{\left(1+r_{k}^{\prime \prime}\right)}
$$

Since $r_{k}^{\prime \prime}<r_{n}^{\prime \prime}$ according to Eq. (5), this subcase condition $\mu(\pi)>1+r_{n}^{\prime \prime}$ implies $\mu(\pi)>1+r_{k}^{\prime \prime}$. This means that $\left[1-\frac{\mu(\pi)}{\left(1+r_{k}^{\prime \prime}\right)}\right]<0$. Also, using Eq. (1), we have $u_{\max }^{n} \geq$ $u_{\max }^{k}$ for $k \leq n$. Therefore, Eq. (31) can be rewritten as:

$$
\begin{aligned}
& \frac{S(\pi)}{\left(1+r_{k}^{\prime \prime}\right)}+u_{\max }^{k}\left[1-\frac{\mu(\pi)}{\left(1+r_{k}^{\prime \prime}\right)}\right] \geq U^{k}-\frac{r_{k}^{\prime \prime} \cdot \mathrm{Q}^{k}}{\left(1+r_{k}^{\prime \prime}\right)} \\
& \equiv \text { (By rearranging) }
\end{aligned}
$$

$$
\left[\frac{S(\pi)-\mu(\pi) \cdot u_{\max }^{k}}{\left(1+r_{k}^{\prime \prime}\right)}+u_{\max }^{k}+\frac{r_{k}^{\prime \prime} \cdot \mathrm{Q}^{k}}{\left(1+r_{k}^{\prime \prime}\right)}\right] \geq U^{k}
$$

Therefore for Subcase (2a), the second condition of Theorem 2 is implied by the premise of this Theorem 3 .

Subcase (2b): In this subcase, we consider $\mu(\pi) \leq 1+r_{n}^{\prime \prime}$ and hence $\delta=u_{\min }^{n}$ according to Eq. (26). From Eq. (30), using $\delta=u_{\min }^{n}$ we have

$$
\begin{aligned}
& \frac{S(\pi)}{\left(1+r_{k}^{\prime \prime}\right)}+u_{\min }^{n}-\frac{\mu(\pi) \cdot u_{\max }^{n}}{\left(1+r_{k}^{\prime \prime}\right)} \geq U^{k}-\frac{r_{k}^{\prime \prime} \cdot \mathrm{Q}^{k}}{\left(1+r_{k}^{\prime \prime}\right)} \\
& \Rightarrow \text { (Using Eq. (1), we have } u_{\max }^{k} \geq u_{\min }^{n} \text { for all } k \leq n \text { ) } \\
& \frac{S(\pi)}{\left(1+r_{k}^{\prime \prime}\right)}+u_{\max }^{k}-\frac{\mu(\pi) \cdot u_{\max }^{n}}{\left(1+r_{k}^{\prime \prime}\right)} \geq U^{k}-\frac{r_{k}^{\prime \prime} \cdot \mathrm{Q}^{k}}{\left(1+r_{k}^{\prime \prime}\right)} \\
& \Rightarrow \text { (Using Eq. 11), we have } u_{\max }^{n} \geq u_{\max }^{k} \text { for all } k \leq n \text { ) } \\
& \frac{S(\pi)}{\left(1+r_{k}^{\prime \prime}\right)}+u_{\max }^{k}-\frac{\mu(\pi) \cdot u_{\max }^{k}}{\left(1+r_{k}^{\prime \prime}\right)} \geq U^{k}-\frac{r_{k}^{\prime \prime} \cdot \mathrm{Q}^{k}}{\left(1+r_{k}^{\prime \prime}\right)} \\
& \equiv \text { (By rearranging) } \\
& {\left[\frac{S(\pi)-\mu(\pi) \cdot u_{\max }^{k}}{\left(1+r_{k}^{\prime \prime}\right)}+u_{\max }^{k}+\frac{r_{k}^{\prime \prime} \cdot \mathrm{Q}^{k}}{\left(1+r_{k}^{\prime \prime}\right)}\right] \geq U^{k}}
\end{aligned}
$$

Therefore for Subcase (2b), the second condition of Theorem 2 is implied by the premise of this Theorem 3 .

We have consequently shown that the first and second condition of Theorem 2 are implied by the premise of this Theorem in Case (1) and in the two subcases of Case (2), respectively. Since the (iterative) sufficient schedulability conditions of Theorem 2 are true whenever the premise of this Theorem is true, the task set $\Gamma^{n}$ is RM schedulable on uniform multiprocessor $\pi$.

\subsection{Schedulability Condition for Unit-Capacity Multiprocessor}

Using the simple sufficient condition of Theorem 3, we can derive a simple sufficient condition for RM schedulability of a task set on the subclass of uniform multiprocessor platforms, called unit-capacity multiprocessors.

Corollary 1. A periodic task system $\Gamma^{n}$ is RM schedulable on an unit-capacity multiprocessor platform $\pi$ having $m$ processors for $m \geq 2$, if

$$
\frac{m\left[1-u_{\max }^{n}\right]}{\left(1+r_{n}^{\prime \prime}\right)}+u_{\max }^{n}+\frac{r_{n}^{\prime} \cdot \mathrm{Q}^{n}}{\left(1+r_{n}^{\prime \prime}\right)} \geq U^{n}
$$


Proof. According to Definition 1 and Definition 2 we have $S(\pi)=m(\pi)=m$ and $\mu(\pi)=m(\pi)=m$, respectively, for unit-capacity multiprocessor platform having $m$ processors (each processor has speed one). Note that, for any task set $\Gamma^{n}$, we also have $r_{n}^{\prime \prime} \leq 1$ according to Eq. (5). Therefore $\left(1+r_{n}^{\prime \prime}\right) \leq 2$, and we consider $m \geq 2$. Hence we have, $m \geq\left(1+r_{n}^{\prime \prime}\right)$ which is equivalent to $\mu(\pi) \geq\left(1+r_{n}^{\prime \prime}\right)$ for unit-capacity multiprocessor platform. Consequently, $\delta=u_{\max }^{n}$ for the value of $\delta$ in the sufficient condition in Eq. 26) of Theorem 3 Substituting the value $S(\pi)=m$, $\mu(\pi)=m$ and $\delta=u_{\max }^{n}$ in the sufficient condition of Eq. (26) of Theorem 3, we have

$$
\begin{gathered}
\frac{S(\pi)-\mu(\pi) \cdot u_{\max }^{n}}{\left(1+r_{n}^{\prime \prime}\right)}+u_{\max }^{n}+\frac{r_{n}^{\prime} \cdot \mathrm{Q}^{n}}{\left(1+r_{n}^{\prime \prime}\right)} \geq U^{n} \\
\equiv \frac{m\left[1-u_{\max }^{n}\right]}{\left(1+r_{n}^{\prime \prime}\right)}+u_{\max }^{n}+\frac{r_{n}^{\prime} \cdot \mathrm{Q}^{n}}{\left(1+r_{n}^{\prime \prime}\right)} \geq U^{n}
\end{gathered}
$$

\section{Performance Comparison}

In this section, we will now measure the improvement of our simple sufficient schedulability conditions for implicitdeadline tasks compared to the corresponding state-of-theart schedulability conditions for global RM scheduling on uniform and unit-capacity multiprocessors.

Uniform Multiprocessors (RM Priority): Prior to this work, the best possible simple (non-iterative) sufficient RM schedulability condition in terms of worst-case system utilization on uniform multiprocessors is derived by Goossens and Baruah in [5] for implicit deadline task set. The schedulability condition from [5] is given in Theorem 4 .

Theorem 4 (Goossens and Baruah [5]). A periodic implicit deadline task system $\Gamma^{n}$ is RM schedulable on an uniform multiprocessor platform $\pi$ if

$$
\frac{S(\pi)-\mu(\pi) \cdot u_{\max }^{n}}{2} \geq U^{n}
$$

The left-hand side of Eq. (33) is smaller than the lefthand side of Eq. 26. Therefore, it is evident that any task set satisfying Eq. (33) must also satisfy Eq. 26), and not necessarily vice versa. In other words, there are some task sets that satisfy Eq. (26) but do not satisfy Eq. (33). Hence, our derived simple sufficient RM schedulability condition given in Theorem 3 dominates the condition in Theorem 4 for uniform multiprocessors.

Unit-Capacity Multiprocessors (RM Priority): Prior to this work, the best possible simple (non-iterative) sufficient RM schedulability condition in terms of worst-case system utilization on unit-capacity multiprocessors is derived by Bertogna, Cirinei and Lipari [8] for implicitdeadline task sets. The schedulability condition from [8] is given in Theorem 5
Theorem 5 (Bertogna, Cirinei, Lipari [8]). An implicitdeadline periodic task system $\Gamma^{n}$ is RM schedulable on a unit-capacity multiprocessor platform having $m$ processors if

$$
\frac{m\left[1-u_{\max }^{n}\right]}{2}+u_{\max }^{n} \geq U^{n}
$$

The left-hand side of Eq. 34 is smaller than the lefthand side of Eq. (32). Hence, our derived simple sufficient RM schedulability condition given in Corollary 1 dominates the condition in Theorem 5 for unit-capacity multiprocessors.

Other Fixed-Priority Scheduling: Prior to this work, the best simple (non-iterative) sufficient fixed-priority (other than RM priority) schedulability condition in terms of worst-case system utilization on unit-capacity multiprocessors is derived by Andersson using the SM-US scheduling algorithm [1] for implicit-deadline task sets. It is proved in [1] that the algorithm SM-US has the worst-case system utilization of $2 m /(3+\sqrt{5}) \approx 38.19 \%$ on $m$ processors.

Our derived simple sufficient RM schedulability condition in Corollary 1 dominates the condition derived in [1] if $u_{\max }^{n} \leq(\sqrt{5}-1) /(3+\sqrt{5})$. This is because if $u_{\max }^{n} \leq(\sqrt{5}-1) /(3+\sqrt{5})$, then the left hand-side of Eq. (32) is at least equal to

$$
\begin{aligned}
& \frac{m[1-(\sqrt{5}-1) /(3+\sqrt{5})]}{\left(1+r_{n}^{\prime \prime}\right)}+u_{\max }^{n}+\frac{r_{n}^{\prime} \cdot \mathrm{Q}^{n}}{\left(1+r_{n}^{\prime \prime}\right)} \\
& \equiv \frac{2}{\left(1+r_{n}^{\prime \prime}\right)} \cdot \frac{2 m}{(3+\sqrt{5})}+u_{\max }^{n}+\frac{r_{n}^{\prime} \cdot \mathrm{Q}^{n}}{\left(1+r_{n}^{\prime \prime}\right)} \geq \frac{2 m}{(3+\sqrt{5})}
\end{aligned}
$$

In general, we can conclude that for other simple fixedpriority schedulability conditions that our simple RM schedulability conditions does not dominate, it is also true that those conditions do not dominate ours either.

Impact of Task-Set Parameters: A main contribution of this paper is the set of task-set parameters that are visible in the schedulability conditions in Theorem 3 and Corollary 1. By analyzing these conditions it can be observed that a positive effect on schedulability occurs in the following cases:

- The sum of squares of individual task utilization is high ( $c f$. parameter $\mathrm{Q}^{n}$ in the numerator of the third term in the condition of Theorem 3 and Corollary 1 ).

- The maximum ratio between periods of any higher and any lower-priority task is small ( $c f$. parameter $r_{n}^{\prime \prime}$ in the denominator of the first and third terms in the condition of Theorem 3 and Corollary 1 .

- The ratio between periods of the highest and the lowest priority task is large ( $c f$. parameter $r_{n}^{\prime}$ in the numerator of the third term in the condition of Theorem 3 and Corollary 1).

A consequence of this is that, if system designers has the freedom to select the parameters of individual tasks (guided by these three strategies), a higher system utilization can be 
achieved compared to a less pro-active (less parameterized) design approach.

Controllability of Task-Set Parameters: The smaller the number of task parameters upon which a condensed task-set parameter depends, the higher is the controllability that task-set parameter provides to the system designer. The task-set parameter minimum period ratio $r_{n}^{\prime}$ depends only on the minimum and the maximum periods of a task set. The maximum period ratio $r_{n}^{\prime \prime}$ depends on all possible pairs of periods of a task set. And the sum of squares of individual task utilization $\mathrm{Q}^{n}$ depends on the WCET and the periods of all the tasks. Therefore, $r_{n}^{\prime}$ provides the highest (and $\mathrm{Q}^{n}$ the lowest) degree of controllability among the three new task-set parameters introduced in the simple RM schedulability conditions in Theorem 3 and in Corollary 1 for uniform and unit-capacity multiprocessors, respectively.

Sensitivity Analysis: We conducted a number of experiments based on the different levels of controllability of the three new task set-parameters to measure their impact on system utilization for unit-capacity multiprocessors. In next subsection, we present our experimental results to estimate the amount of improvement of our simple schedulability test for unit-capacity processors in corollary 1 over the simple schedulability test for that of in Theorem 5 The conclusions from the experiments are that they not only corroborate the performance predictions made above but also show order-of-magnitude performance improvements (in terms of number of scheduled task sets) for carefullyselected (i.e. controlled) task-set parameters.

\subsection{Experimental Evaluation}

We have theoretically proved the dominance of our schedulability test in Corollary 1 (we call P J test ${ }^{2}$ ) over the schedulability test in Theorem 5 (called BCL test) for unitcapacity-processors. To quantitatively estimate the degree of dominance of the PJ test over the BCL test, a series of experiments are conducted using randomly generated task sets.

\subsubsection{Simulation Setup}

We run a number of 36 experiments. Each experiment has five simulation parameters: $m, \min U, \max U$, $T_{\max }$ and $T_{\min }$. Each experiment is characterized by the value of $m$, the range $(\min U, \max U]$ and the integer set $\left\{T_{\min }, \ldots T_{\max }\right\}$. The value of $m$ denotes the number of processors we consider for an experiment. Each experiment is carried out for a number of randomly generated task sets. The utilization $u_{i}$ of a randomly generated task $\tau_{i}$ of a task set is uniformly distributed within $(\min U, \max U]$. The

\footnotetext{
${ }^{2}$ The name of the test is given by concatenating the first characters of the authors' last names
}

period $T_{i}$ of the task $\tau_{i}$ is randomly selected from the set $\left\{T_{\min }, \ldots T_{\max }\right\}$. The worst case execution time $C_{i}$ of task $\tau_{i}$ is then $U_{i} \times T_{i}$.

The number of processors we consider in our experiments are $m=2,4,6$, and 8 . This parameter $m$ is used to measure the impact of increasing number of processors (scalability) on schedulability test. We consider three different utilization ranges $(0,0.5],(0.25,0.75]$ and $(0,1]$ for $(\min U, \max U]$. The utilization ranges $(0,0.5],(0.25,0.75]$ and $(0,1]$ are used to experiment with light, medium and mixed ${ }^{3}$ tasks, respectively. In order to see the impact of periods of a task set on the schedulability test, three different period sets $\{100, \ldots 1000\}$, $\{500, \ldots 1000\}$ and $\{750, \ldots 1000\}$ are considered for $\left\{T_{\min }, \ldots T_{\max }\right\}$. The four values of $m$, the three utilization ranges for $(\min U, \max U]$ and the three period-sets for $\left\{T_{\min }, \ldots T_{\max }\right\}$ constitute our 36 different experiments.

For each experiment, a total of 100000 task sets are randomly generated such that each of the 100000 task sets pass the P J test in corollary 1 The 100000 task sets are generated according to the following procedure:

1. Initially, we generate $m+1$ tasks.

2. Then we verify if the generated task set passes the P J test.

3. If the answer of the PJ test is positive, then it is counted as one of the 100000 task sets. Then, we verify the BCL test for this task set. And,

(a) if the answer for the BCL test is positive, then this task set passes both the P J and BCL tests.

(b) if the answer for the BCL test is negative, then this task set only passes the PJ test and fails to pass the BCL test.

(c) then by adding one new task, we extend this (old) task set to a new task set and return to Step 2

4. If the answer of the $\mathrm{P} J$ test is negative, then we discard this task set and go to Step 1.

For each experiment, the total number of task sets (of the 100000 task sets that pass $\mathrm{PJ}$ test) that pass the BCL test is counted in variable $\mathrm{BCL}_{\text {count }}$. The dominance factor of the PJ test, denoted by $D_{P J}$, is given using Eq. (35) for each experiment.

$$
\mathrm{D}_{\mathrm{PJ}}=\frac{100000-\mathrm{BCL}_{\text {count }}}{100000} \times 100 \%
$$

The higher the value of $\mathrm{D}_{\mathrm{PJ}}$, the higher is the dominance of the $\mathrm{P} J$ test over the BCL test. For example, if the dominance factor $D_{P_{J}}=20 \%$, then $20 \%$ (20000 task sets) of the 100000 task sets are not schedulable by the BCL test.

\footnotetext{
${ }^{3}$ mixed task includes heavy tasks in addition to light and medium tasks
} 


\subsubsection{Simulation Result}

The result of the 36 experiments are grouped into three categories (each category has 12 experiments) based on the three different sets for $\left\{T_{\min }, \ldots T_{\max }\right\}$. The result of the 12 experiments in each of the three categories are given in Table 1 Table 2 and Table 3 for $\left\{T_{\min }, \ldots T_{\max }\right\}$ equals to $[100, \ldots 1000],[500, \ldots 1000]$ and $[750, \ldots 1000]$, respectively. Each of the shaded cells in Table 1 Table 2 and

Table 1. Value of $\mathrm{D}_{\mathrm{PJ}}$ for the 12 experiments using $\left\{T_{\min } \ldots T_{\max }\right\}=\{100 \ldots 1000\}$

\begin{tabular}{|c|c|c|c|}
\hline & \multicolumn{3}{|c|}{$(\min U, \max ]$} \\
\hline & $(0,1]$ & $(0,0.5]$ & $(0.25,0.75]$ \\
\hline$m=2$ & $21.42 \%$ & $15.56 \%$ & $67.14 \%$ \\
\hline$m=4$ & $16.94 \%$ & $11.12 \%$ & $63.48 \%$ \\
\hline$m=6$ & $16.74 \%$ & $10.46 \%$ & $63.50 \%$ \\
\hline$m=8$ & $16.20 \%$ & $10.30 \%$ & $63.32 \%$ \\
\hline
\end{tabular}

Table 2. Value of $D_{P J}$ for the 12 experiments using $\left\{T_{\min } \ldots T_{\max }\right\}=\{500 \ldots \mathbf{1 0 0 0}\}$

\begin{tabular}{|l|l|c|c|}
\hline & \multicolumn{3}{|c|}{$(\min U, \max U]$} \\
\hline & $(0,1]$ & $(0,0.5]$ & $(0.25,0.75]$ \\
\hline$m=2$ & $20.18 \%$ & $16.92 \%$ & $63.74 \%$ \\
\hline$m=4$ & $23.80 \%$ & $17.08 \%$ & $73.80 \%$ \\
\hline$m=6$ & $29.56 \%$ & $21.28 \%$ & $81.24 \%$ \\
\hline$m=8$ & $35.3 \%$ & $24.52 \%$ & $87.46 \%$ \\
\hline
\end{tabular}

Table 3. Value of $\mathrm{D}_{\mathrm{PJ}}$ for the 12 experiments using $\left\{T_{\min } \ldots T_{\max }\right\}=\{750 \ldots 1000\}$

\begin{tabular}{|c|c|c|c|}
\hline & \multicolumn{3}{|c|}{$(\min U, \max ]$} \\
\hline & $(0,1]$ & $(0,0.5]$ & $(0.25,0.75]$ \\
\hline$m=2$ & $21.06 \%$ & $18.08 \%$ & $63.92 \%$ \\
\hline$m=4$ & $27.28 \%$ & $22.08 \%$ & $79.28 \%$ \\
\hline$m=6$ & $37.02 \%$ & $27.98 \%$ & $88.26 \%$ \\
\hline$m=8$ & $45.48 \%$ & $31.96 \%$ & $93.46 \%$ \\
\hline
\end{tabular}

Table 3 represents the value of $\mathrm{D}_{\mathrm{PJ}}$ for simulation parameters - number of processors and utilization range - given in the corresponding first column and second row of each table, respectively.

\subsubsection{Result Analysis}

Observe that the P J test in Corollary 1 provides better RM schedulability (higher system utilization) if minimum period ratio $r_{n}^{\prime}$ is large, maximum period ratio $r_{n}^{\prime \prime}$ is small and/or sum of square of utilization of individual task utilization $\mathrm{Q}^{n}$ is large.

The impact of utilization ranges $(\min U, \max U]$ on the dominance of $\mathrm{P} J$ test over $\mathrm{BCL}$ test is significant. The value of dominance, that is $\mathrm{D}_{\mathrm{PJ}}$, for the $\mathrm{PJ}$ test over the $\mathrm{BCL}$ test using medium tasks (i.e. $u_{i}$ is within $[0.25,0.75]$ ) is significantly higher (see the forth columns of each Table) than that of using light and mixed tasks. The BCL test fails to schedule more than $63 \%$ task sets of the 100000 task sets that are schedulable by the PJ test. This is because of the use of the task-set parameter sum of square (i.e. $\mathrm{Q}^{n}$ ) in the $\mathrm{P} J$ test. Each task $\tau_{i}$ adds $u_{i}^{2}$ (except $u_{\max }^{n}{ }^{2}$ ) to the calculation of $\mathrm{Q}^{n}$ according to Eq. (6). Since all tasks are medium, the value of $u_{i}^{2}>0.25^{2}=0.0625$. The higher the value of $u_{i}$, the higher is the value of $\mathrm{Q}^{n}$ and the higher is the system utilization. Consequently, the value of $\mathrm{D}_{\mathrm{PJ}}$ is comparatively much higher using medium tasks than that of using light or mix tasks. In summary, PJ tests significantly outperforms $B C L$ tests for medium tasks.

The relationship of the periods of a task set has varying impacts on the dominance factor of the P $\mathrm{J}$ test over the BCL test. Observe that, according to Eq. (2) the minimum period ratio $r_{n}^{\prime}$ of a task set is the ratio between the minimum and maximum periods (i.e. one pair of periods) of a task set. As a result when the periods of the tasks of a task set are randomly selected from a set of denser integer values, then the value of $r_{n}^{\prime}$ can be expected to be higher than that of when the periods are selected from a set of dispersed integer values. The values of $\mathrm{D}_{\mathrm{P} J}$ in most of the shaded cells in Table 3 are larger than that of the corresponding cells in either Table 1 or Table 2 since the task periods in Table 3 are selected from a denser set $\{750 \ldots 1000\}$ than that of in either Table 1 or Table 2

The value of maximum period ratio $r_{n}^{\prime \prime}$ depends on the number of tasks, number of processors and the set $\left\{T_{\min } \ldots T_{\max }\right\}$ that are used for selecting the periods of the randomly generated tasks of a task set. Recall that, according to Eq. (3), the maximum period ratio $r_{n}^{\prime \prime}$ of a task set depends on all different pairs of periods of a task set. For a set of $n$ tasks, there are $\left(\begin{array}{l}n \\ 2\end{array}\right)=\frac{n !}{(n-2) ! \cdot 2 !}$ different pairs of periods. The higher is the number of tasks in a task set, the higher is the number of different pairs of periods. The higher is the number of different pairs of periods, the higher is the possibility that the values of two periods are closer. And the closer the two periods are, the higher is the value of maximum period ratio $r_{n}^{\prime \prime}$ (according to Eq. (3)). Also note that the way tasks are generated for a task set implies that the higher is the number of processors, the higher is the 
number of tasks and hence the higher is the probability that the value of $r_{n}^{\prime \prime}$ is larger. And according to Eq. (6), the value of $\mathrm{Q}^{n}$ increases as more tasks are included in a task set. Therefore, as we increase the number of processors, both the values of $r_{n}^{\prime \prime}$ and $\mathrm{Q}^{n}$ increases.

Each of the 12 experimental results in Table 1 are derived based on tasks' periods that are selected from a set of relatively dispersed integer values. Therefore, we expect the value of minimum period period $r_{n}^{\prime}$ of each task set to be relatively small. As we increase the number of processors even though the value of $\mathrm{Q}^{n}$ increases (positive impact factor on schedulability), the smaller value of $r_{n}^{\prime}$ and larger value of $r_{n}^{\prime \prime}$ (negative impact factors on schedulability) result in decreasing value of dominance of the PJ test over $\mathrm{BCL}$ test for all the three utilization ranges (see the decreasing trend in each column of Table 1). The negative impact factors (i.e. low $r_{n}^{\prime}$ and high $r_{n}^{\prime \prime}$ ) can not be offset by the positive impact factor (i.e. high $\mathrm{Q}^{n}$ ) for the simulation parameters used to generate the values given in Table 1 . Therefore, as we increase the number of processors (i.e. increase in number of tasks in a task set), the value of $D_{P J}$ decreases for all types (light, medium and mixed) of tasks.

However, the dominance factor increases as we increase the number of processors for each of the utilization ranges as shown in Table 2 and Table 3 This is because even though the value of $r_{n}^{\prime \prime}$ increases (negative impact factor on schedulability) as we increase the number of processors, the value of $\mathrm{Q}^{n}$ increases and the value of $r_{n}^{\prime}$ is relatively larger for using a relatively denser set for periods. The negative impact of the higher value of $r_{n}^{\prime \prime}$ is offset by the positive impact of the higher value of $r_{n}^{\prime}$ and $\mathrm{Q}^{n}$. Consequently, as we increase the number of processors, the value of $\mathrm{D}_{\mathrm{PJ}}$ increases for all three utilization ranges for Table 2 and Table 3 . In summary, if the ratio of the minimum and maximum period of a task set are closer (value of $r_{n}^{\prime}$ is larger), then the PJ test scales well with increasing number of processors for any kind (light, medium and mix) of task sets.

\section{Conclusion}

In this paper we have proposed schedulability conditions for global rate-monotonic scheduling of implicit-deadline tasks on multiprocessors. The prominent feature of our conditions is that they introduce a set of new task-set parameters that are key to achieving good schedulability performance in terms of worst-case system utilization. The two proposed simple RM schedulability conditions for uniform and unit-capacity processors are shown to dominate any other known simple RM schedulability condition. For other simple fixed-priority schedulability conditions that our conditions does not dominate, it is shown that those conditions do not dominate our schedulability condition either.

\section{References}

[1] B. Andersson. Global Static-Priority Preemptive Multiprocessor Scheduling with Utilization Bound 38\%. in Proc. of Prin. of Dist. Syst., pages 73-88, 2008.

[2] B. Andersson, S. Baruah, and J. Jonsson. Static-Priority Scheduling on Multiprocessors. in Proc. of RTSS, pages 193-202, 2001.

[3] B. Andersson and J. Jonsson. The utilization bounds of partitioned and pfair static-priority scheduling on multiprocessors are 50\%. in Proc. of ECRTS, pages 33-40, 2003.

[4] T. P. Baker. An Analysis of Fixed-Priority Schedulability on a Multiprocessor. Real-Time Systems, 32(1-2):49-71, 2006.

[5] S. Baruah and J. Goossens. Rate-Monotonic Scheduling on Uniform Multiprocessors. IEEE Trans. on Comput., 52(7):966-970, 2003.

[6] S. Baruah and J. Goossens. Deadline Monotonic Scheduling on Uniform Multiprocessors. in Proc. of Prin. of Dist. Syst., pages 89-104, 2008.

[7] O. Beaumont, L. Arnaud, and Y. Robert. Static Scheduling Strategies for Heterogeneous Systems. Computing and Informatics, 21:413-430, 2002.

[8] M. Bertogna, M. Cirinei, and G. Lipari. New Schedulability Tests for Real-Time Task Sets Scheduled by Deadline Monotonic on Multiprocessors . in Proc. of Prin. of Dist. Syst., pages 306-321, 2005.

[9] M. Bertogna, M. Cirinei, and G. Lipari. Schedulability Analysis of Global Scheduling Algorithms on Multiprocessor Platforms. IEEE Trans. on Parallel and Dist. Syst., 20(4):553-566, 2009.

[10] E. Bini, T. H. C. Nguyen, P. Richard, and S. Baruah. A Response-Time Bound in Fixed-Priority Scheduling with Arbitrary Deadlines. IEEE Trans. Comput., 58(2):279-286, 2009.

[11] S. Chaudhry, R. Cypher, M. Ekman, M. Karlsson, A. Landin, S. Yip, H. Zeffer, and M. Tremblay. Rock: A HighPerformance Sparc CMT Processor. IEEE Micro, 29(2):616, 2009.

[12] R. I. Davis and A. Burns. Priority Assignment for Global Fixed Priority Pre-Emptive Scheduling in Multiprocessor Real-Time Systems. In Proc. of RTSS, pages 398-409, 2009.

[13] S. K. Dhall and C. L. Liu. On a Real-Time Scheduling Problem. Operations Research, 26(1):127-140, 1978.

[14] S. Funk, J. Goossens, and S. Baruah. On-Line Scheduling on Uniform Multiprocessors. in Proc. of RTSS, pages 183-192, 2001.

[15] T. Gonzalez, O. H. Ibarra, and S. Sahni. Bounds for LPT Schedules on Uniform Processors. SIAM Journal on Computing, 6(1):155-166, 1977.

[16] J. Goossens, S. Funk, and S. Baruah. Priority-Driven Scheduling of Periodic Task Systems on Multiprocessors. Real-Time Systems., 25(2-3):187-205, 2003.

[17] H. Jin and P. Tan. A Novel Dynamic Allocation and Scheduling Scheme with CPNA and FCF Algorithms in Distributed Real-time Systems. In Proc. of ICPADS, pages 550-556, 2005.

[18] C. L. Liu and J. W. Layland. Scheduling Algorithms for Multiprogramming in a Hard-Real-Time Environment. Journal of the ACM, 20(1):46-61, 1973.

[19] J. M. López, M. García, J. L. Díaz, and D. F. García. Utilization Bounds for Multiprocessor Rate-Monotonic Scheduling. Real-Time Systems, 24(1):5-28, 2003. 
[20] L. Lundberg. Analyzing Fixed-Priority Global Multiprocessor Scheduling. in Proc. of RTAS, pages 145-153, 2002.

[21] D.-I. Oh and T. P. Baker. Utilization Bounds for N-Processor Rate Monotone Scheduling with Static Processor Assignment. Real-Time Systems, 15(2):183-192, 1998.

[22] L. Sha, J. P. Lehoczky, and R. Rajkumar. Solutions for Some Practical Problems in Prioritized Preemptive Scheduling. In Proc. of RTSS, pages 181-191, 1986. 